\title{
RE: Universal Tumor DNA BRCA1/2 Testing of Ovarian Cancer: Prescreening PARPi Treatment and Genetic Predisposition
}

\author{
Federica Tomao, Pierluigi Benedetti Panici, Silverio Tomao
}

Correspondence to: Silverio Tomao, MD, Dipartimento Scienze Radiologiche, Oncologiche e Anatomo Patologiche Sapienza University of Rome, Viale Regina Elena 324, Rome, Italy (e-mail: silverio.tomao@gmail.com).

BRCA1/2 mutations play a predictive role in ovarian cancer risk evaluation. Moreover, patients are today being tested for BRCA1/2 mutations to select a tailored therapy because they could benefit from a treatment with PARP inhibitors (PARPis). Therefore, in ovarian carcinomas (OCs), BRCA1/2 mutation testing is an important step in planning the correct therapeutic strategy in association with chemotherapy and anti-vascular endothelial growth factor agents. We read with great interest the recent article in the Journal by Vos et al. (1) that investigates the role of universal tumor DNA BRCA1/2 testing of all newly diagnosed OC patients as prescreen for PARPi treatment and cancer predisposition. With the approach described by the authors, hereditary as well as somatic aberrations affecting DNA BRCA1/ 2 could be quickly and correctly detected with tumor BRCA1/2 single-molecule molecular inversion, probe-based, next-generation sequence testing. The authors concluded that this test, as the first step in all newly diagnosed OC patients, could statistically significantly increase the identification rate of eligible patients for treatment with PARPis. Even if this result can contribute to modifying clinical practice, it is appropriate to make some observations.

According to Vos et al. (1), all 315 patients were not distinguished according to histotype (generically ovarian cancer patients were recruited and investigated). This is a considerable gap if one only considers that in women unselected for family history, germline BRCA1/2 mutations have been found in 4-14\% of all OCs, $5-18 \%$ of serous OCs, and approximately $22 \%$ of highgrade serous cases (2). The Cancer Genome Atlas consortium detected BRCA1/2 mutations in $20 \%$ of 316 high-grade serous cases; $9 \%$ and $8 \%$ were germline BRCA1 and BRCA2 mutations, respectively, and $3 \%$ were somatic mutations (3). OC DNA testing was performed according to methodology described by
Weren et al. (4). According to Weren et al. (4), it is not specified whether some parameters had been evaluated to ensure good quality of formalin-fixed, paraffin-embedded samples for DNA analysis (as the recommendations for information to be included when reporting tumor BRCA1/2 results), according to Capoluongo et al. (2). In the section describing the study population, Vos et al. (1) declare that in few cases were cells obtained from ascites aspiration analyzed. Even if there are only a few cases, it should be pointed out that, at present, there are no mature data supporting the use of the ascitic liquid as a source of cells to carry out mutational analysis of BRCA1/2 in OC. Tissue samples from primary carcinomas and metastases, formalinfixed, paraffin-embedded, and fresh-frozen specimens, constitute the most suitable source of material to carry out DNA BRCA1/2 testing $(2,5)$. Vos et al. (1) declared also that universal BRCA1/2 testing is well appreciated by patients and gynecologists. Undoubtedly, this statement is correct and shareable, but we must not forget that only 13 of 17 patients consented to the telephone interview and only 18 of 41 gynecologists compiled and delivered the evaluation questionnaire. These numbers are decidedly poor to allow us to draw firm conclusions on the compliance and satisfaction of patients and doctors toward the test.

\section{Notes}

Dipartimento Materno Infantile e Scienze Urologiche, Sapienza University of Rome (FT, PBP); Dipartimento Scienze Radiologiche, Oncologiche e Anatomo Patologiche Sapienza University of Rome (ST).

The authors declare no conflict of interest in preparing and submitting this manuscript. 


\section{References}

1. Vos JR, Fakkert IE, de Hullu JA, et al. Universal tumor DNA BRCA1/2 testing of ovarian cancer: prescreening PARPi treatment and genetic predisposition. J Natl Cancer Inst. 2020;112(2):161-169.

2. Capoluongo E, Ellison G, López-Guerrero JA, et al. Guidance statement on BRCA1/2 tumor testing in ovarian cancer patients. Semin Oncol. 2017;44(3): 187-197. doi:10.1053/j.seminoncol.2017.08.004
3. Cancer Genome Atlas Research Network. Integrated genomic analysis of ovarian carcinoma. Nature. 2011;474(7353):609-615.

4. Weren RD, Mensenkamp AR, Simons $M$, et al. Novel BRCA1 and BRCA2 tumor test as basis for treatment decisions and referral for genetic counselling of patients with ovarian carcinomas. Hum Mutat. 2017;38(2):226-235.

5. Vergote I, Banerjee S, Gerdes AM, et al. Current perspectives on recommendations for BRCA genetic testing in ovarian cancer patients. Eur J Cancer. 2016;69: 127-134. 